\title{
Resveratrol effectively attenuates $\alpha$-naphthyl- isothiocyanate-induced acute cholestasis and liver injury through choleretic and anti-inflammatory mechanisms
}

\author{
Tao WANG ${ }^{1,3}$, Zhi-xing ZHOU ${ }^{2}$, Li-xin SUN ${ }^{1}$, Xia LI ${ }^{1}$, Zhi-meng XU ${ }^{1}, \mathrm{Mi} \mathrm{CHEN}^{1}$, Guo-lin ZHAO ${ }^{1}$, Zhen-zhou JIANG ${ }^{1,3, *}$, \\ Lu-yong ZHANG ${ }^{1,4, *}$ \\ ${ }^{1}$ Jiangsu Center for Drug Screening, China Pharmaceutical University, Nanjing 210009, China; ${ }^{2}$ Tianjin Key Laboratory of \\ Molecular Design and Drug Discovery, Tianjin Institute of Pharmaceutical Research, Tianjin 300193, China; ${ }^{3} \mathrm{Jiangsu}$ Center for \\ Pharmacodynamics Research and Evaluation, China Pharmaceutical University, Nanjing 210009, China; ${ }^{4}$ State Key Laboratory of \\ Natural Medicines, China Pharmaceutical University, Nanjing 210009, China
}

\begin{abstract}
Aim: $\alpha$-Naphthylisothiocyanate (ANIT) is a well-characterized cholestatic agent for rats. The aim of this study was to examine whether resveratrol could attenuate ANIT-induced acute cholestasis and liver injury in rats.

Methods: SD rats were treated with resveratrol (15 or $30 \mathrm{mg} / \mathrm{kg}$, ip) or a positive control drug ursodeoxycholic acid (100 mg/kg, po) for 5 consecutive days followed by a single dose of ANIT $(60 \mathrm{mg} / \mathrm{kg}, \mathrm{po})$. Bile flow, and serum biochemical markers and bile constituents were measured $48 \mathrm{~h}$ after ANIT administration. Hepatic levels of oxidative repair enzymes (glutathione peroxidase, catalase and MnSOD), myeloperoxidase activity, TNF- $\alpha$, IL-6, and ATP content, as well as the expression of liver transporter genes and proteins were assayed.

Results: ANIT exposure resulted in serious cholestasis and liver injury, as shown by marked neutrophil infiltration in liver, dramatically increased serum levels of ALT, AST, GGT, ALP, TBA, TBIL, IBIL, and DBIL, and significantly decreased bile excretion and biliary output of GSH and $\mathrm{HCO}_{3}{ }^{-}$. ANIT significantly increased TNF- $\alpha$ and IL-6 release and myeloperoxidase activity, decreased mitochondrial biogenesis in liver, but had little effect on hepatic oxidative repair enzymes and ATP content. Furthermore, ANIT significantly decreased the expression of Mrp2, FXR, and Cyp7a1, markedly increased Mrp3 expression in liver. Pretreatment with resveratrol attenuated ANITinduced acute cholestasis and liver injury, and other pathological changes. Pretreatment with ursodeoxycholic acid was less effective. Conclusion: Resveratrol effectively attenuates ANIT-induced acute cholestasis and liver injury in rats, possibly through suppression of neutrophil infiltration, as well as upregulation of expression of hepatic transporters and enzymes, thus decreasing accumulation of bile acids.
\end{abstract}

Keywords: resveratrol; ursodeoxycholic acid; $\alpha$-naphthylisothiocyanate; cholestasis; liver injury; cytokine; bile acids; hepatic injury; cytokine; bile acids; hepatic transporters; FXR; Cyp7a1

Acta Pharmacologica Sinica (2014) 35: 1527-1536; doi: 10.1038/aps.2014.119; published online Nov 242014

\section{Introduction}

Cholestasis results in a dramatic increase in liver and serum bile acid levels that eventually leads to acute liver toxicity, proliferation of bile ducts, and fibrosis that progresses to cirrhosis $^{[1]}$. Cholestasis is often divided into two categories (intrahepatic or extrahepatic) based on its etiology. Bile salts

\footnotetext{
* To whom correspondence should be addressed.

E-mail lyzhang@cpu.edu.cn (Lu-yong ZHANG); jiangcpu@163.com (Zhen-zhou JIANG)

Received 2014-08-25 Accepted 2014-10-10
}

are crucial components of cholestasis and are potentially toxic to living cells. Early studies on the mechanisms of cholestatic liver injury strongly implicated bile acid-induced apoptosis as the major cause of hepatocellular injury. Recent work has focused on the role of both bile acids in cell signaling and sterile inflammation in the pathophysiology of cholestasis. Thus, reducing the intracellular content and cytotoxicity of bile acids (and other potentially toxic cholephilic compounds that accumulate after secretory failure) is very important for the prevention of cholestatic liver injury ${ }^{[2-4]}$.

To decrease the accumulation of unconjugated bile acids 
inside hepatocytes and alleviate acute cholestasis, the liver compensates through the adaptive downregulation of hepatic uptake transporters and upregulation of efflux transporters. These modifications can last for a few days or weeks before liver failure or other serious liver injuries occur ${ }^{[5-7]}$. To halt the process of liver fibrosis, early intervention for cholestasis is critical. These compensatory processes provide a suitable window for the treatment of cholestasis. A number of alternative drugs are currently being tested in pre-clinical studies as potential treatments for cholestatic disease, including selective modulators of nuclear receptors and signaling pathways that are thought to mediate cholestasis ${ }^{[8,9]}$.

Resveratrol (3,5,4'-trans-trihydroxystilbene), a polyphenol phytoalexin abundantly found in grape skins and wine, possesses diverse biochemical and physiological actions. Resveratrol has been shown to significantly increase Sirtuin (SIRT) activity, an $\mathrm{NAD}^{+}$(oxidized form of nicotinamide adenine dinucleotide)-dependent histone deacetylase. Peroxisome proliferators-activated receptor (PPAR) gamma co-activator1alpha (PGC-1a) is a member of a family of transcriptional coactivators that regulate mitochondrial biogenesis, antioxidation, growth factor signaling, and angiogenic activity. Resveratrol has been shown to increase PGC-1a functions in vivo by increasing SIRT1 activity ${ }^{[10]}$. Current studies indicate that resveratrol exerts its pharmacological preconditioning and stress resistance by activating PGC-1a in tissues with high oxidative capacities, including brown fat, heart, kidney, skeletal muscle and brain ${ }^{[11]}$.

Recently, it was found that resveratrol improved cholestatic liver injury by decreasing fibrosis and promoting hepatocyte regeneration ${ }^{[12]}$, decreasing oxidative damage ${ }^{[13]}$, and modulating apoptosis, mitochondrial biogenesis and autophagy ${ }^{[14]}$. The current evidence for the protective effect of resveratrol on cholestasis was mainly obtained from studies using bile duct ligation (an extrahepatic model) in animals. However, the effect of resveratrol on rats with intrahepatic cholestasis remains unclear.

The adaptive regulation of hepatobiliary transporter expression has been proposed to reduce liver injury during cholestasis ${ }^{[15,16]}$. Although the protective effects of resveratrol on cholestasis have been described, little is known about the role of resveratrol in modulating liver bile acid transporters and enzyme systems during cholestasis. In the present study, intrahepatic cholestasis was modeled by the administration of a-naphthylisothiocyanate (ANIT), a well-characterized cholestatic agent, to rats ${ }^{[17]}$. We then sought to determine whether resveratrol reduced ANIT-induced acute cholestasis and liver injury in rats by altering the levels of liver bile acid transporters.

\section{Materials and methods Materials}

Resveratrol, ANIT, and ursodeoxycholic acid (UDCA) were obtained from Sigma Chemical Co (St Louis, MO, USA). Corn oil, Tween-20 and other reagents were obtained from Wanqing Chemical Industry Ltd (Nanjing, China) and used without further purification. Sodium taurocholate cotransporting polypeptide (Ntcp) rabbit polyclonal antibody (sc-98485), multidrug-resistant-associated protein 3 (Mrp3) rabbit polyclonal antibody (sc-20767), multidrug-resistant-associated protein 2 (Mrp2) mouse monoclonal antibody (sc-59611), bile salt export pump (Bsep) rabbit polyclonal antibody (sc-25571), farnesoid $X$ receptor (FXR) rabbit polyclonal antibody (sc-13063), cholesterol 7a-hydroxylase (Cyp7a1) rabbit polyclonal antibody (sc25536), $\beta$-actin mouse monoclonal antibody (sc-19879), and an ECL chemiluminescence kit were obtained from Santa Cruz Biotechnology Inc (Dallas, Texas, USA). HRP-conjugated secondary antibodies were obtained from KPL Inc (CA, USA).

\section{Animals and drug treatments}

All experiments were performed according to the regulations of the administration of affairs concerning experimental animals (Ministry of Science, China, 1998) and approved by the Ethics Committee of China Pharmaceutical University (Nanjing). Forty 12-week old male Sprague-Dawley rats were purchased from SIPPR-BK Experimental Animal Company (SPF Grade, Shanghai, China) and housed in a humidity- and temperature-controlled environment with a 12-h light/12-h dark cycle. The rats received a standard diet and water ad libitum. After 1 week of acclimation, the rats were randomly divided into 5 experimental groups. Group I consisted of control rats that received intraperitoneal (ip) injections of the vehicle (saline) for five consecutive days followed by an intragastric (ig) administration of corn oil (vehicle of ANIT). Group II consisted of ANIT-treated rats that received the vehicle and ig administration of ANIT (dissolved in corn oil) at a dose of $60 \mathrm{mg} / \mathrm{kg}$ body weight, after the last vehicle injection. Group III consisted of ANIT plus UDCA rats that were given an oral administration of UDCA at a dose of $100 \mathrm{mg} / \mathrm{kg}$ body weight for five consecutive days prior to administration of ANIT (60 $\mathrm{mg} / \mathrm{kg}$, ig) $30 \mathrm{~min}$ after the last UDCA administration. UDCA was suspended in $0.5 \%$ carboxymethylcellulose sodium solution. Groups IV and V consisted of ANIT plus resveratrol rats that were intraperitoneally administered resveratrol at a dose of 15 or $30 \mathrm{mg} / \mathrm{kg}$ body weight for five consecutive days prior to the administration of ANIT $(60 \mathrm{mg} / \mathrm{kg}$, ig) $30 \mathrm{~min}$ after the last injection.

\section{Experimental procedures}

All animals were treated according to the schedule outlined above. At $48 \mathrm{~h}$ after the initial ANIT or vehicle treatment, $0.5 \mathrm{~mL}$ of blood was collected from each rat from the orbital venous plexus under ether anesthesia after an 8 -h fast. The animals were anesthetized with urethane $(1 \mathrm{~g} / \mathrm{kg}$, ip) after the last blood collection, and a middle abdominal incision was made to cannulate the common bile duct (PE-10, Intramedic, Clay Adams Parsippany, NJ, USA). To measure bile flow and analyze bile composition, bile samples were collected for $2 \mathrm{~h}$. During this time, the body temperature of the animals was maintained with a heat pad to prevent hypothermic alterations in the bile flow. At the end of each experiment, the animals were euthanized by exsanguination, and the liver was 
immediately removed and weighed. The liver, serum, and bile samples obtained were stored at $-80^{\circ} \mathrm{C}$ until use.

\section{Serum biochemistry}

Serum alanine aminotransferase (ALT), aspartate aminotransferase (AST), y-glutamyl transpeptidase (GGT), alkaline phosphatase (ALP), total bilirubin (TBIL), direct bilirubin (DBIL), indirect bilirubin (IBIL) and total bile acid (TBA) were assayed using a commercially available clinical test kit with a biochemistry analyzer system (Synchron Clinical System Lx 20; Beckman Coulter Inc, Fullerton, CA, USA).

\section{Collection of bile and determination of bile constituents}

Rats were anesthetized with urethane. Next, cannulations of the common bile duct were performed ${ }^{[18]}$. After a 3-min equilibration, bile was collected for $2 \mathrm{~h}$ to determine the content of bile acid, phospholipids, bilirubin, glutathione (GSH) and $\mathrm{HCO}_{3}{ }^{-}$secretion, as well as the bile output and flow rate. Bile density was calculated by accurately weighing $100 \mu \mathrm{L}$ of the bile; bile flow was determined by gravimetry. The output of biliary components was calculated as the product between bile flow and biliary concentration. The contents of bile acids, phospholipids and bilirubin were determined using an Automatic Clinical Analyzer (7080, HITACHI Ltd, Tokyo, Japan). The concentration of GSH in the bile was measured by colorimetry $(405 \mathrm{~nm})$ after reaction with 5,5'-dithiobis-(2-nitrobenzoic acid). $\mathrm{HCO}_{3}{ }^{-}$concentrations were calculated from $\mathrm{pH}$ and $\mathrm{pCO}_{2}$ using the Henderson-Hasselbach equilibrium equation. $\mathrm{pH}$ and $\mathrm{pCO}_{2}$ were measured immediately after bile collection in an automated blood gas analyzer (Radiometer ABL800, Radiometer Medical Ltd., Broenshoej, Denmark).

\section{Assays of hepatic components and enzymes}

Livers were homogenized in nine volumes of ice-cold normal saline. The homogenate was centrifuged at $4{ }^{\circ} \mathrm{C}(4000 \mathrm{r} / \mathrm{min}$, $20 \mathrm{~min}$ ), and the resultant supernatant was used for assays of hepatic components and enzymes.

The activity of glutathione peroxidase (GPx), catalase and manganese superoxide dismutase (MnSOD) was determined to evaluate the oxidative status in the liver tissue. The hepatic catalase, MnSOD and GPx activities were determined using their respective activity assay kits (Nanjing Jiancheng Bioengineering Institute, Nanjing, China).

Myeloperoxidase (MPO) activity and the levels of tumor necrosis factor alpha (TNF- $\alpha$ ) and interleukin-6 (IL-6) were determined to assess neutrophil activation and inflammatory reactions, respectively. The hepatic MPO activity was determined using a commercial assay kit (Nanjing Jiancheng Bioengineering Institute, Nanjing, China). The TNF- $\alpha$ and IL-6 levels from homogenates were determined using an ELISA kit (UNIV-bio, Shanghai, China).

As mitochondria are a primary source of ATP, ATP content was determined using the Kinase-Glo ${ }^{\circledast}$ Max Luminescent Kinase Assay kit (Promega, USA) as a basis for mitochondrial function.

\section{Determination of hepatic mtDNA copy number}

DNA samples were extracted from liver tissue. The mitochondrial DNA (mtDNA) copy number was then measured by real-time PCR and corrected by simultaneous measurement of the nuclear DNA. The forward and reverse primers complementary to the nuclear $\beta$-actin gene were: $5^{\prime}$-GAAATCGTGCGTGACATTAAAG-3' and 5'-ATCGGAACCGCTCATTG-3', respectively. The forward and reverse primers for mtDNA, which are complementary to the sequence of the mitochondrial COX1 gene, were 5'-GGAGCAGTATTCGCCATCAT-3' and 5'-CGGCCGTAAGTGAGATGAAT-3', respectively. The PCR was performed in an IQ5 Real-Time PCR System (BioRad Co, USA) as previously described ${ }^{[14]}$. The threshold cycle number $(\mathrm{Ct})$ values of the $\beta$-actin gene and the mitochondrial ND1 gene were determined for each individual quantitative $\mathrm{PCR}$ run. The $\mathrm{Ct}$ values were used as a measure of the input copy number, and differences in the $\mathrm{Ct}$ values were used to quantify mtDNA copy number relative to the $\beta$-actin gene with the following equation: Relative copy number $(\mathrm{Rc})=2^{\Delta \mathrm{CT}}$, where $\Delta \mathrm{CT}$ was the $\mathrm{Ct}_{\beta \text {-actin }}-\mathrm{Ct}_{\mathrm{mtDNA}}$. Each measurement was carried out at least three times and normalized within each experiment against a serial dilution of a control DNA sample.

\section{Liver transporter gene expression using real-time PCR}

TRIzol reagent (Invitrogen Life Technologies, Carlsbad, CA) was used to prepare RNA. An aliquot of $1 \mu \mathrm{g}$ of total RNA from each sample was reverse-transcribed (RT) to cDNA using PrimeScript ${ }^{\mathrm{TM}}$ Reverse Transcriptase (Takara, Osaka, Japan). Quantification of gene expression was performed using a realtime polymerase chain reaction (PCR) kit (Takara, Osaka, Japan), and reports were generated using the $\mathrm{iQ}^{\mathrm{TM}} 5$ Optical System Software (version 2.1, BioRad). GAPDH was used for internal normalization. Primers for the genes Ntcp, Mrp2, Mrp3, Bsep, Cyp7a1, FXR, and GAPDH were synthesized (Sunshine Biotechnology, Nanjing, China) using the sequences listed in Table 1 . The fidelity of the PCR reaction was determined by melting temperature analysis.

\section{Western blot analysis}

Liver protein extraction for the analysis of Ntcp, Mrp2, Mrp3, Bsep, FXR, and Cyp7a1 levels ( $n=8$ in each group) was performed using a total protein extraction kit (keyGEN Biotech, Nanjing, China). The protein concentrations of the extracts were determined using a BCA Protein Assay reagent kit (Beyotime Biotech, Nanjing, China). An equal amount of total protein $(10 \mu \mathrm{L})$ per lane was fractionated on an $8 \%$ SDSpolyacrylamide gel. After electrophoresis, the gels were transferred onto polyvinylidene difluoride membranes, which were then blocked with Tris-buffered saline containing 5\% nonfat dry milk at $4^{\circ} \mathrm{C}$. Next, the membranes were incubated overnight at $4{ }^{\circ} \mathrm{C}$ in a solution containing $0.1 \%$ Tween $20,5 \%$ nonfat dry milk, and a dilution of the antibody of interest: antiNtcp (1:300), anti-Bsep (1:300), anti-Mrp2 (1:100), anti-Mrp3 (1:500), anti-FXR (1:300), anti-Cyp7a1 (1:500), or anti- $\beta$-actin (1:500). After three washes in TBS-T, the membranes were 
Table 1. Primer sequences of six genes selected for real-time RT-PCR with GAPDH as an internal control.

\begin{tabular}{ll}
\hline Gene name & \multicolumn{1}{c}{ Primers $\left(5^{\prime}-3^{\prime}\right)$} \\
\hline \multirow{2}{*}{ Ntcp } & F: GCATGATGCCACTCCTCTTATAC \\
& R: TACATAGTGTGGCCTTTTGGACT \\
Mrp2 & R: CAGTCACGGCTTCCTTTCTG \\
& F: ACACCGATCCCACTGGGACTTCT \\
Mrp3 & R: TCAGCTTCACATTGCCTATC \\
& F: TGGAAAGGAATGGTATGGG \\
Bsep & R: CAGAAGGCCAGTGCATAACACA \\
& F: AGGCCATGTTCCTTCGTTA \\
FXR & R: TTCAGCTCCCCGACACTTTT \\
& F: TGGATCAAGTGCAACTGAATGAC \\
Cyp7a1 & R: GCACTGGAAAGCCTCAGAGC \\
& F: ATGGAGAAGGCTGGGGCTCACCT \\
GAPDH & R: AGCCCTTCCACGATGCCAAAGTTG \\
&
\end{tabular}

F, forward primer; R, reverse primer.

incubated with HRP-conjugated secondary antibodies at room temperature for $1 \mathrm{~h}$ and subsequently processed for enhanced chemiluminescence (ECL) detection using a Super Signal Substrate kit (Pierce Chemical, Rockford, IL, USA). Signals were detected using a chemiluminescence system (Bio-Rad, California, USA). Samples were assessed for $\beta$-actin content as an internal control. The relative expression of target proteins was calculated as the grayscale ratio of the target protein to that of $\beta$-actin ${ }^{[19]}$. Each experimental group was tested three times.

\section{Histological examination}

Rat liver samples were taken from the central part of the right large lobe from both the control and ANIT-exposed groups at $48 \mathrm{~h}$ post-intoxication. The liver samples were fixed with $10 \%$ formalin in phosphate-buffered saline for $24 \mathrm{~h}$ and then washed with tap water, dehydrated in alcohol, and embedded in paraffin. Sections ( $5 \mu \mathrm{m}$ thick) were mounted on glass slides. Histological changes were investigated after staining with hematoxylin and eosin (H\&E) by a senior pathologist in a blinded manner. Histological lesions were graded by analyzing the portal or lobular inflammation and necrosis as follows: grade 0 , no change; grade 1 , minimal portal inflammation with minimal or patchy lobular inflammation and necrosis; grade 2 , mild portal inflammation with mild lobular inflammation and little hepatocellular change; grade 3 , moderate portal inflammation, moderate lobular inflammation and necrosis with noticeable hepatocellular damage; and grade 4 , severe portal and lobular inflammation, necrosis with prominent diffuse hepatocellular damage.

\section{Statistical analysis}

Data were analyzed using the PASW statistics package version 18 (SPSS Inc, Chicago, IL, USA) and represented as the mean $\pm S D$. The significance of differences between groups was evaluated using Student's $t$-test. Differences were considered significant at $P<0.05$.

\section{Results \\ Effect of resveratrol on serum biochemical markers}

When compared with the vehicle group, ANIT intoxication significantly increased the levels of serum ALT, AST, GGT, ALP, TBA, TBIL, IBIL, and DBIL, thus resulting in serious liver injury and cholestasis. Resveratrol at dosages of either 15 or $30 \mathrm{mg} / \mathrm{kg}$ body weight significantly reversed the majority of ANIT-induced increases in the above parameters. The lower dose of resveratrol slightly improved TBA and IBIL levels. UDCA significantly decreased ALT, AST, and ALP but had little effect on the other markers (Table 2).

\section{Effect of resveratrol on bile flow and biliary secretions}

ANIT decreased bile flow at $48 \mathrm{~h}$ after administration; furthermore, resveratrol and UDCA significantly attenuated this change (Figure 1). Biliary output of TBA, phospholipids, TBIL, DBIL, GSH, and $\mathrm{HCO}_{3}{ }^{-}$were significantly decreased after ANIT exposure. Resveratrol and UDCA significantly increased their output (Table 3).

\section{Effect of resveratrol on antioxidant enzymes}

The hepatic activities of catalase, GPx and MnSOD were mildly decreased at $48 \mathrm{~h}$ after ANIT administration in rats. These changes were not statistically significant compared with the control group. Antioxidant enzyme activity was unchanged after treatment with resveratrol or UDCA (data not shown).

Table 2. Effects of pretreatment with resveratrol or UDCA on the serum biomarkers $48 \mathrm{~h}$ after ANIT administration in rats. Control: vehicle only; Model: ANIT $60 \mathrm{mg} / \mathrm{kg}$, ig; UDCA: UDCA (100 mg/kg, ig)+ANIT (60 mg/kg, ig); Resv-H: resveratrol (30 mg/kg, ip)+ANIT (60 mg/kg, ig); Resv-L: resveratrol (15 $\mathrm{mg} / \mathrm{kg}, \mathrm{ip})+$ ANIT $\left(60 \mathrm{mg} / \mathrm{kg}\right.$, ig). Mean \pm SEM. $n=8 .{ }^{\mathrm{c}} P<0.01$ vs control group. ${ }^{\mathrm{e}} P<0.05,{ }^{\mathrm{f}} P<0.01$ vs model group.

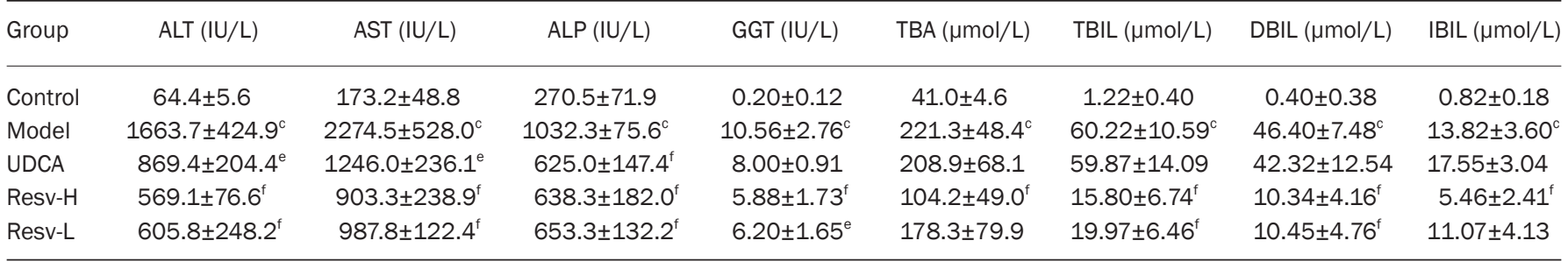




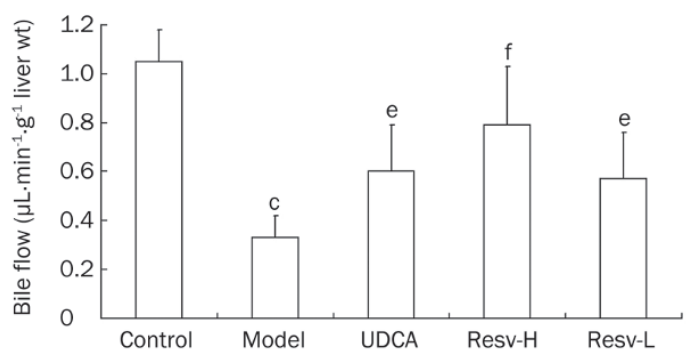

Figure 1. Effects of pretreatment with resveratrol or UDCA on basal bile flow $48 \mathrm{~h}$ after ANIT administration in rats. Bile specimens were collected $2 \mathrm{~h}$ at $48 \mathrm{~h}$ after treatment with ANIT. Control: vehicle only; Model: ANIT $60 \mathrm{mg} / \mathrm{kg}$, ig; UDCA: UDCA (100 mg/kg, ig)+ANIT (60 mg/kg ig); Resv-H: resveratrol (30 mg/kg, ip)+ANIT (60 mg/ $\mathrm{kg}$, ig); Resv-L: resveratrol (15 $\mathrm{mg} / \mathrm{kg}$, ip)+ANIT (60 mg/kg, ig). Mean \pm SEM. $n=8 .{ }^{\mathrm{b}} P<0.05,{ }^{\mathrm{c}} P<0.01 \mathrm{vs}$ control group. ${ }^{\mathrm{e}} P<0.05,{ }^{\mathrm{f}} \mathrm{P}<0.01$ vs model group.

\section{Effect of resveratrol on hepatic MPO activity and inflammatory cytokines}

It is well known that inflammation is involved in cholestasis. Our results showed that hepatic MPO activity and the levels of TNF- $\alpha$ and IL- 6 were significantly increased at $48 \mathrm{~h}$ after ANIT intoxication. Both doses of resveratrol significantly attenuated the ANIT-induced increases in hepatic MPO activity and TNF- $\alpha$ and IL-6 levels, indicating that resveratrol exerts potent anti-inflammatory effects. UDCA produced mild decreases in both hepatic MPO activity and levels of TNF-a and IL-6 (Figure 2).

\section{Effect of resveratrol on hepatic ATP content and mtDNA copy number}

Previous reports have shown that exposure to high levels of hydrophobic bile acids can eventually lead to mitochondrial dysfunction, ATP depletion and cell death in cholestasis. Unexpectedly, we observed that the hepatic ATP content was slightly increased at $48 \mathrm{~h}$ after ANIT intoxication compared with the control group. Both doses of resveratrol mildly decreased hepatic ATP content, but the results did not reach statistically significant differences versus the control group. Additionally, UDCA had no effect on hepatic ATP content (Figure 3). The mtDNA copy number was measured to verify the activation of mitochondrial biogenesis. The mtDNA copy
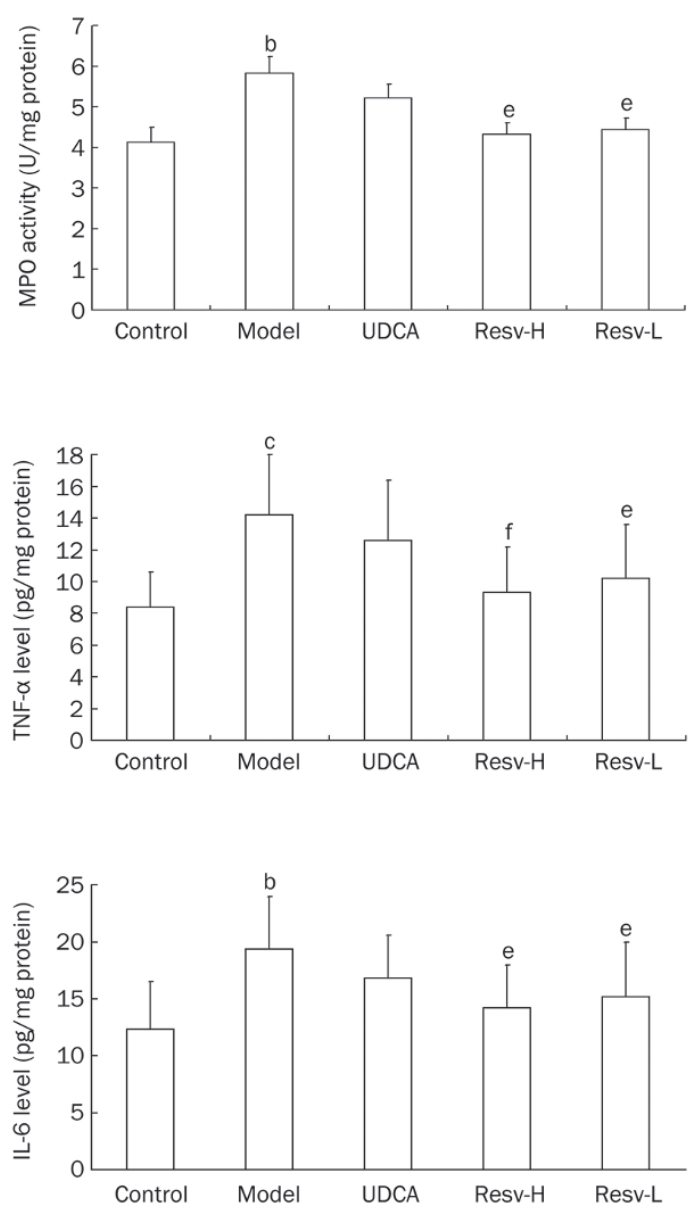

Figure 2. Effects of pretreatment with resveratrol or UDCA on hepatic myeloperoxidase (MPO) activity and TNF- $\alpha$ and IL-6 levels at $48 \mathrm{~h}$ after ANIT administration in rats. Control: vehicle only; Model: ANIT $60 \mathrm{mg} / \mathrm{kg}$, ig; UDCA: UDCA (100 mg/kg, ig)+ANIT (60 mg/kg, ig); Resv-H: resveratrol (30 mg/kg, ip)+ANIT (60 mg/kg, ig); Resv-L: resveratrol $(15 \mathrm{mg} / \mathrm{kg}$, ip)+ANIT (60 mg/kg, ig). Mean \pm SEM. $n=8 .{ }^{b} P<0.05,{ }^{c} P<0.01$ vs control group. ${ }^{\mathrm{e}} P<0.05,{ }^{\mathrm{f}} P<0.01$ vs model group.

number was significantly decreased at $48 \mathrm{~h}$ after ANIT intoxication; however, this change was not reversed by either resveratrol or UDCA treatment (Figure 3).

Table 3. Effects of pretreatment with resveratrol or UDCA on biliary components secretion $48 \mathrm{~h}$ after ANIT administration in rats. Control: vehicle only; Model: ANIT 60 mg/kg, ig; UDCA: UDCA (100 mg/kg, ig)+ANIT (60 mg/kg, ig); Resv-H: resveratrol (30 mg/kg, ip)+ANIT (60 mg/kg, ig); Resv-L: resveratrol (15 mg/kg, ip)+ANIT (60 mg/kg, ig). Mean \pm SEM. $n=8 .{ }^{b} P<0.05,{ }^{c} P<0.01$ vs control group. ${ }^{e} P<0.05,{ }^{f} P<0.01$ vs model group.

\begin{tabular}{|c|c|c|c|c|c|c|}
\hline Group & $\begin{array}{c}\text { TBA output } \\
\left(\mu \mathrm{mol} \cdot \mathrm{min}^{-1} \cdot \mathrm{g}^{-1} \text { liver } \mathrm{wt}\right)\end{array}$ & $\begin{array}{l}\text { Phospholipids output } \\
\text { (nmol.min }{ }^{-1} \cdot g^{-1} \text { liver wt) }\end{array}$ & $\begin{array}{c}\text { TBIL output } \\
\left(\mathrm{nmol} \cdot \mathrm{min}^{-1} \cdot \mathrm{g}^{-1} \text { liver wt }\right)\end{array}$ & $\begin{array}{c}\text { DBIL output } \\
\left(\mathrm{nmol} \cdot \mathrm{min}^{-1} \cdot \mathrm{g}^{-1} \text { liver wt }\right)\end{array}$ & $\begin{array}{c}\text { GSH output } \\
\left(\mathrm{nmol} \cdot \mathrm{min}^{-1} \cdot \mathrm{g}^{-1} \text { liver wt }\right)\end{array}$ & $\begin{array}{c}\mathrm{HCO}_{3}^{-} \text {output } \\
\text { (nmol.min'-1. } \mathrm{g}^{-1} \text { liver wt) }\end{array}$ \\
\hline Control & $7.4 \pm 1.3$ & $7.3 \pm 1.2$ & $15.2 \pm 2.8$ & $10.7 \pm 3.5$ & $1.21 \pm 0.11$ & $26.4 \pm 1.8$ \\
\hline UDCA & $2.1 \pm 0.4^{f}$ & $6.2 \pm 1.6^{\mathrm{e}}$ & $54.8 \pm 16.9^{f}$ & $50.1 \pm 20.4^{f}$ & $1.29 \pm 0.11^{\mathrm{e}}$ & $16.9 \pm 2.2^{\mathrm{e}}$ \\
\hline Resv-H & $2.7 \pm 1.3^{f}$ & $7.8 \pm 1.8^{f}$ & $53.2 \pm 12.7^{f}$ & $49.7 \pm 39.2^{f}$ & $1.37 \pm 0.16^{f}$ & $22.3 \pm 2.5^{f}$ \\
\hline Resv-L & $1.9 \pm 0.4^{f}$ & $5.7 \pm 1.4$ & $41.8 \pm 7.3^{f}$ & $39.9 \pm 17.1^{f}$ & $1.17 \pm 0.17^{e}$ & $11.5 \pm 0.4$ \\
\hline
\end{tabular}



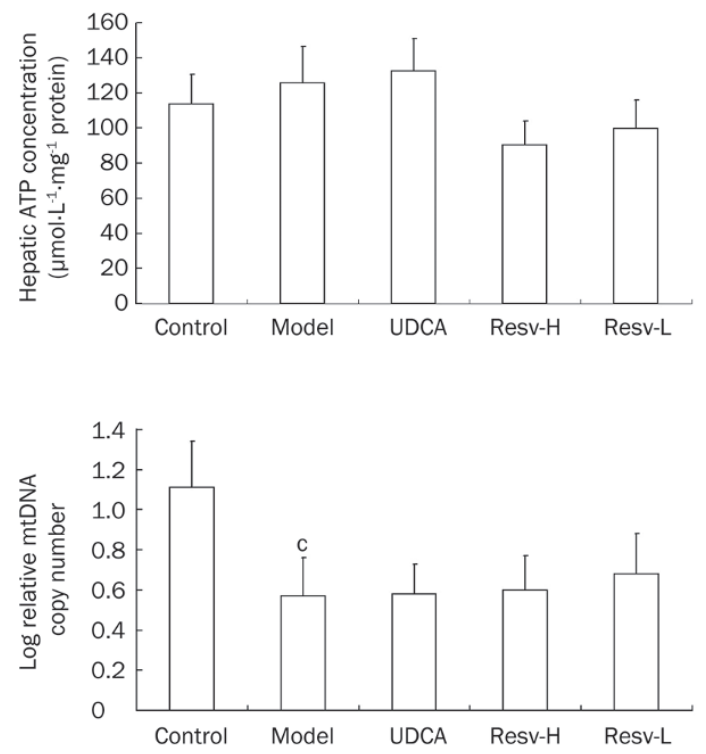

Figure 3. Effects of pretreatment with resveratrol or UDCA on hepatic mitochondrial DNA (mtDNA) copy number and ATP concentration at $48 \mathrm{~h}$ after ANIT administration in rats. mtDNA was corrected by the amount of nuclear DNA. Control: vehicle only; Model: ANIT $60 \mathrm{mg} / \mathrm{kg}$, ig; UDCA: UDCA (100 mg/kg, ig)+ANIT (60 mg/kg, ig); Resv-H: resveratrol (30 $\mathrm{mg} / \mathrm{kg}$, ip)+ANIT (60 mg/kg, ig); Resv-L: resveratrol (15 mg/kg, ip)+ANIT (60 mg/kg, ig). Mean \pm SEM. $n=8 .{ }^{b} P<0.05,{ }^{c} P<0.01$ vs control group. ${ }^{\mathrm{e}} \mathrm{P}<0.05,{ }^{\mathrm{f}} \mathrm{P}<0.01$ vs model group.

Effect of resveratrol on hepatic bile acid transporter expression Because adaptive regulation has been proposed as a mechanism to limit hepatotoxicity, we quantified the mRNA and protein levels of uptake and efflux transporters in rats after ANIT exposure. Forty-eight hours after ANIT administration, the mRNA levels of Ntcp, Mrp2, and Bsep were decreased by $34 \%, 62 \%$, and 36\%, respectively; in contrast, Mrp3 mRNA showed a 3-fold increase. The mRNA levels of FXR and Cyp7a1 were decreased by $77 \%$ and $44 \%$, respectively. Both doses of resveratrol enhanced Mrp2, FXR, and Cyp7a1 mRNA expression and significantly inhibited Mrp3 mRNA expression. Resveratrol did not, however, affect Ntcp and Bsep mRNA expression. Meanwhile, UDCA significantly enhanced Mrp2 mRNA expression but had little effect on the other markers (Figure 4A).

In accordance with mRNA levels, the protein levels of Ntcp, Mrp2, Bsep, FXR, and Cyp7a1 were decreased and Mrp3 was increased $48 \mathrm{~h}$ after ANIT administration. Both doses of resveratrol increased Mrp2, FXR, and Cyp7a1 protein levels, decreased Mrp3 protein expression but had little effect on the protein expression of either Ntcp or Bsep (Figure 4B).

\section{Histological examination}

Liver sections were stained with $H \& E$ and examined for necrosis and inflammation at $48 \mathrm{~h}$ after intoxication. The control group showed few histological changes, but the ANITintoxicated group showed multifocal periportal coagulative necrosis and degenerative changes with an accumulation of neutrophils and monocytes. Resveratrol (30 mg/kg) co-administration clearly attenuated neutrophil and monocyte infiltration as well as ANIT-induced necrotic and degenerative changes. The effects of resveratrol at $15 \mathrm{mg} / \mathrm{kg}$ were similar but slightly weaker compared with the $30 \mathrm{mg} / \mathrm{kg}$ dose (Figure 5). Histologic grading indicated significant amelioration in those animals treated with resveratrol (Table 4). These histological improvements were also found after UDCA treatment (100 mg/kg).

\section{Discussion}

There are certainly additional beneficial properties of resveratrol that have yet to be discovered; in addition, the mechanisms behind some of the best-established therapeutic properties of resveratrol require further examination. The present
A

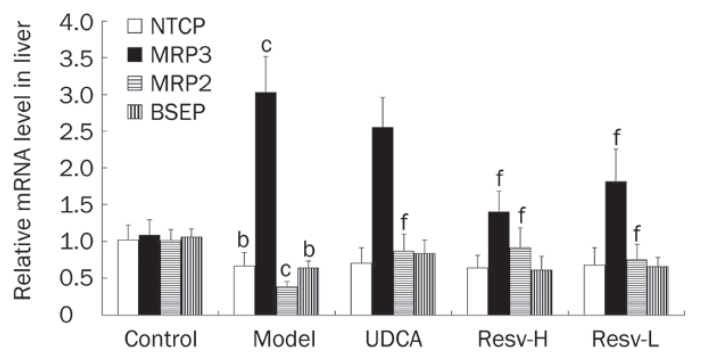

B

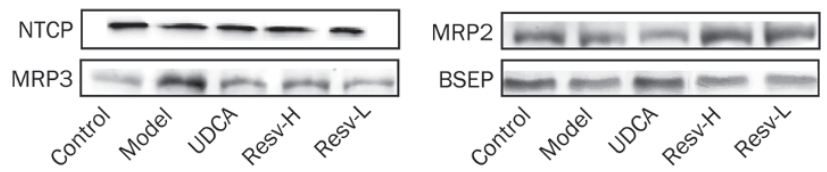

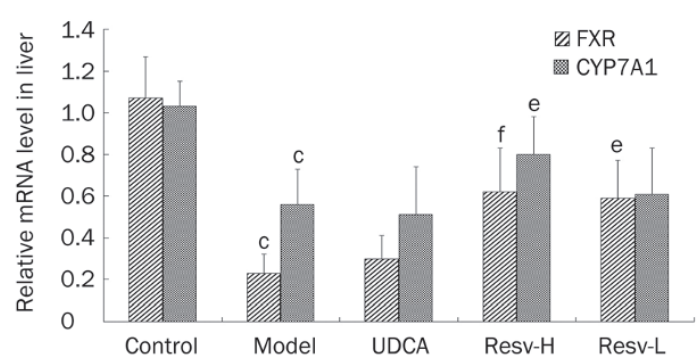
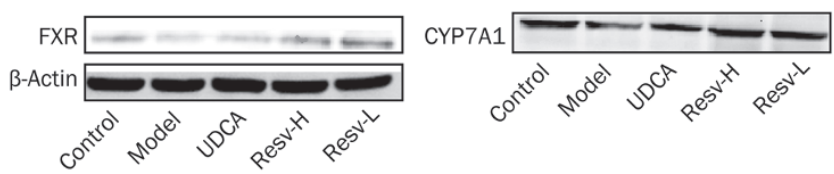

Figure 4. Effects of pretreatment with resveratrol or UDCA on hepatic mRNA expression of uptake and efflux transporters $48 \mathrm{~h}$ after ANIT administration in rats. Control: vehicle only; Model: ANIT $60 \mathrm{mg} / \mathrm{kg}$, ig; UDCA: UDCA (100 mg/kg, ig)+ANIT (60 mg/kg, ig); Resv-H: resveratrol (30 mg/kg, ip)+ANIT (60 $\mathrm{mg} / \mathrm{kg}$, ig); Resv-L: resveratrol (15 mg/kg, ip)+ANIT (60 mg/kg, ig). Mean \pm SEM. $n=8 .{ }^{b} P<0.05,{ }^{\mathrm{C}} P<0.01$ vs control group. ${ }^{\mathrm{e}} P<0.05,{ }^{\mathrm{f}} P<0.01 \mathrm{vs}$ model group. 

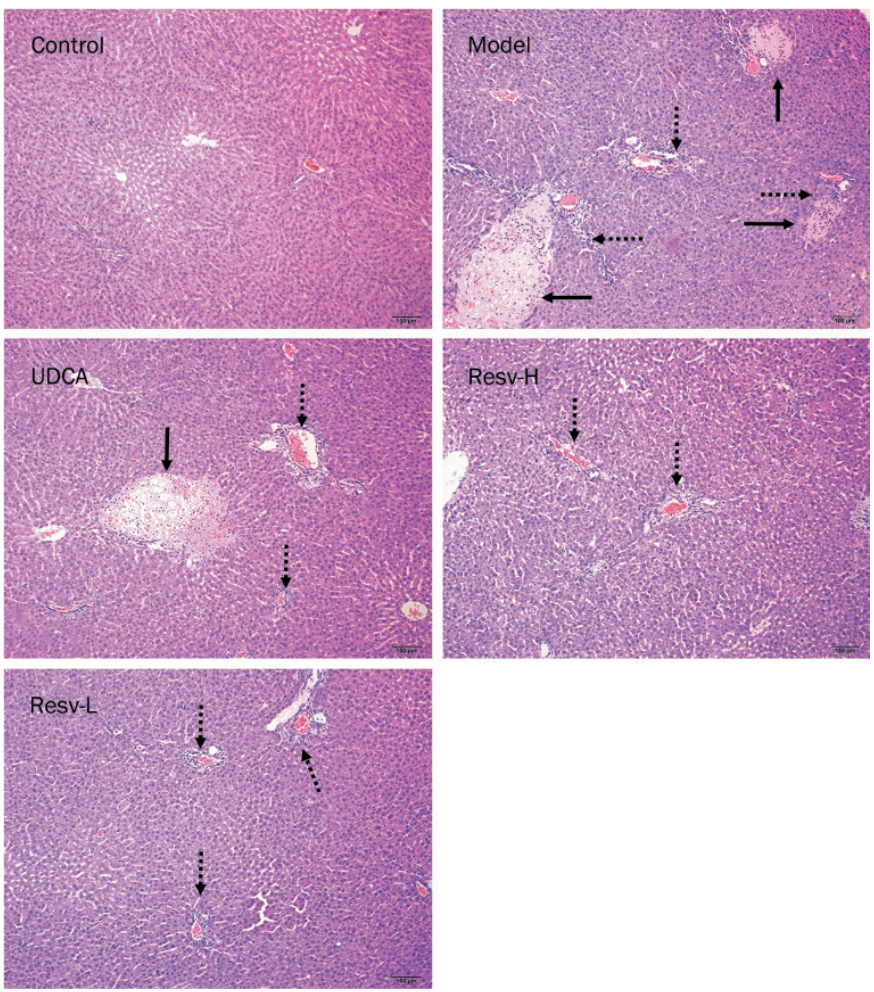

Figure 5. Histological figures of liver cells from control rats and $\alpha$-naphthylisothiocyanate (ANIT)-intoxicated rats with or without pretreatment $(\times 100)$. Control: vehicle only, little histological change was observed; Model: ANIT $60 \mathrm{mg} / \mathrm{kg}$, ig, necrotic and degenerative changes with severe inflammatory cell infiltration were observed; UDCA: UDCA $(100 \mathrm{mg} / \mathrm{kg}$, ig)+ANIT (60 mg/kg, ig), necrotic and degenerative changes with moderate inflammatory cell infiltration were observed; Resv-H: resveratrol (30 $\mathrm{mg} / \mathrm{kg}$, ip)+ANIT (60 mg/kg, ig), little necrotic and degenerative changes and mild to moderate inflammatory cell infiltration were observed; Resv-L: resveratrol (15 mg/kg, ip)+ANIT (60 mg/kg, ig), little necrotic and degenerative changes and mild to moderate inflammatory cell infiltration were observed. $n=8$. Solid arrow, necrosis; dashed arrow, inflammation.

scientific efforts have focused on taking advantage of the specific molecular events that occur in different disease states to design more 'personalized' therapeutic strategies. Thus, we used ANIT in rats to model intrahepatic cholestasis and then sought to determine whether resveratrol plays a protective and/or adaptive role in this type of liver injury ${ }^{[2,21]}$. Markers for liver injury and cholestasis, such as serum ALT, AST, ALP, GGT, bile acids and bilirubin, were increased $24 \mathrm{~h}$ (data not shown) after ANIT administration and peaked at $48 \mathrm{~h}$ (Table 2). These data are in agreement with a previous report ${ }^{[22]}$. Therefore, we assessed the effects of ANIT exposure $48 \mathrm{~h}$ after administration for our present study. An ANIT dosing regimen was adopted as described previously ${ }^{[23]}$. Because of variable resveratrol dosage schemes and its reported poor oral bioavailability, we administered resveratrol at doses of 15 and $30 \mathrm{mg} \cdot \mathrm{kg}^{-1} \cdot \mathrm{d}^{-1}$ via intraperitoneal injections, which were reported to alleviate rat extrahepatic cholestasis and oxidative damage $e^{[12,14]}$.
Table 4. Effects of pretreatment with resveratrol or UDCA on histologic grade of liver lesions at $48 \mathrm{~h}$ after ANIT administration in rats. Control: vehicle only; Model: ANIT 60 mg/kg, ig; UDCA: UDCA (100 mg/kg, ig)+ANIT (60 mg/kg, ig) ; Resv-H: resveratrol (30 mg/kg, ip)+ANIT (60 mg/kg, ig); Resv-L: resveratrol (15 mg/kg, ip)+ANIT (60 mg/kg, ig). Mean \pm SEM. $n=8$. ${ }^{b} P<0.05,{ }^{c} P<0.01$ vs control group. ${ }^{e} P<0.05,{ }^{f} P<0.01$ vs model group.

\begin{tabular}{cc}
\hline Group & Histologic grade $(0-4)$ \\
\hline Control & $0.0 \pm 0.0$ \\
Model & $2.5 \pm 0.5^{\mathrm{c}}$ \\
UDCA & $1.6 \pm 0.7^{\mathrm{e}}$ \\
Resv-H & $1.1 \pm 0.4^{\dagger}$ \\
Resv-L & $1.5 \pm 0.5^{\dagger}$ \\
\hline
\end{tabular}

Grading is based on microscopic evaluation of liver sections by analysis of portal or lobular inflammation and necrosis: grade 0 , no change; grade 1 , minimal portal inflammation with minimal or patchy lobular inflammation and necrosis; grade 2, mild portal inflammation with mild lobular inflammation and little hepatocellular change; grade 3 , moderate portal inflammation, moderate lobular inflammation and necrosis with noticeable hepatocellular damage; and grade 4, severe portal and lobular inflammation, necrosis with prominent diffuse hepatocellular damage.

Resveratrol dose-dependently altered biochemical serum markers of liver damage and cholestasis, such as ALT, AST, ALP, GGT, bile acids and bilirubin, as well as bile flow and bile salt output. Histological examination also indicated obvious improvements in necrosis and inflammation at $48 \mathrm{~h}$ after ANIT intoxication following resveratrol treatment 30 and $15 \mathrm{mg} / \mathrm{kg}$ ). Taken together, resveratrol displayed significant hepatoprotective effects due to a reduction in the intracellular content/cytotoxicity of bile acids and other potentially toxic cholephilic compounds that accumulated after secretory obstruction.

Oxidative injury likely plays a large role in ANIT-induced biliary epithelial injury, possibly as a result of one or more sulfhydryl-reactive intermediates created by repeated rounds of secretion and reuptake of the glutathione conjugate ${ }^{[20,24]}$. Mitochondria are an important source of reactive oxygen species, particularly after cholestasis, when their antioxidant properties are exhausted ${ }^{[25]}$. Indeed, reactive oxygen species can attack mitochondrial membrane phospholipids, DNA and proteins, uncoupling oxidative phosphorylation and altering the capacity for ATP synthesis ${ }^{[26]}$. In this study, liver oxidative repair enzymes (including GPx, catalase and MnSOD) and ATP synthesis remained largely unchanged; however, the liver mtDNA copy number was significantly decreased at $48 \mathrm{~h}$ after ANIT intoxication. Taken together, our results indicate that the impairment of mitochondrial biogenesis, rather than oxidative injury and mitochondrial oxidative phosphorylation, is a critical event at this stage of ANIT-induced cholestasis. The activity of antioxidant enzymes, ATP synthesis and mtDNA copy number remained unchanged after resveratrol treatment in ANIT-intoxicated rats, implying that the antioxidant properties and mitochondrial protection of resveratrol 
did not significantly contribute to the observed improvement of acute cholestasis.

The release of cytotoxic and inflammatory mediators, including those that attract neutrophils, has been suggested to contribute to the hepatic injury induced by ANIT; however, the exact mechanisms are not well understood ${ }^{[27-29]}$. In the present study, an enhancement in the hepatic activity of MPO, an index of tissue neutrophil infiltration, occurred after ANITinduced liver injury. These data confirm previously reported results $^{[30,31]}$. Resveratrol administered intraperitoneally to ANIT-treated rats after the appearance of liver injury significantly reduced the increase in hepatic MPO activity. These results suggest that intraperitoneally administered resveratrol exerts a preventive effect on ANIT-induced liver injury in rats by inhibiting neutrophil infiltration into the liver tissue. Moreover, resveratrol is known to protect against ischemia/ reperfusion-induced liver injury in rats by inhibiting neutrophil infiltration and release of cytokines such as TNF- $\alpha$, IL-1 $\beta$, and IL- $6^{[32]}$. We observed a suppression of liver TNF- $a$ and IL-6 levels in the present study, suggesting that resveratrol decreases the early release of cytokines and neutrophil recruitment, leading to a reduction in late inflammatory processes and an improvement in liver injury after ANIT intoxication.

In agreement with previous studies, we found that ANIT affected the bile salt-independent fraction (BSIF) of the bile flow $^{[22]}$. This was due, in part, to impairments in the biliary output of both GSH and $\mathrm{HCO}_{3}^{-}$, the two major determinants of the BSIF. Our results showed that resveratrol markedly counteracted the effect of ANIT on BSIF, based on the normalization of GSH output and a partial restoration of $\mathrm{HCO}_{3}{ }^{-}$output. The effects of ANIT on biliary secretion of GSH have not been completely elucidated. ANIT is conjugated with GSH in hepatocytes and transported into the bile by multidrug resistance-associated protein (Mrp) 2 (Abcc2), the canalicular efflux transporter. The ANIT-GSH conjugate dissociates upon crossing the canalicular membrane, yielding free GSH and ANIT in the bile ${ }^{[20,33]}$. In this study, the biliary secretions of GSH were extensively increased by resveratrol, which may contribute to the secretion of ANIT. A lack of $\mathrm{HCO}_{3}{ }^{-}$-rich bile secreted at the ductular level may concentrate potentially toxic biliary constituents, thus contributing to cholangiocyte damage in many chronic cholestatic diseases. ANIT treatment also decreased $\mathrm{HCO}_{3}{ }^{-}$biliary output; however, resveratrol only partially counteracted this effect. This beneficial effect likely accelerates the elimination of ANIT by promoting bile flow and attenuating ANIT-induced cholestasis.

At the basolateral membrane of hepatocytes, Ntcp transports bile acids and other substrates from the portal blood supply into hepatocytes. Mrp3-mediated efflux allows for the conjugation of bile acids and xenobiotics in the blood. At the canalicular membrane of hepatocytes, Bsep and Mrp2 transport bile acids into the bile. Mrp2 also transports GSH and conjugated bilirubin. The expression of uptake and efflux transporters is altered during various forms of liver injury. In general, the expression of uptake transporters is reduced, and the levels of efflux transporters are increased in damaged livers ${ }^{[34,35]}$. This adaptive response is thought to reduce the intracellular concentration of bile acids and other toxicants in an attempt to protect against further injury while promoting cellular recovery. Based on existing reports, ANIT has wideranging effects on liver basolateral and canalicular transporters $^{[23]}$. Recently, a report demonstrated that ANIT could directly induce the retention of bile acids in hepatocytes by inhibiting the function of bile acid transporters, which might contribute to its cholestatic effect ${ }^{[36]}$. Our results showed that ANIT significantly decreased the Ntcp, Bsep and Mrp2 levels and increased the Mrp3 levels in the liver $48 \mathrm{~h}$ after administration. Both doses of resveratrol attenuated the changes in Mrp2 and Mrp3 but did not alter the protein levels of Ntcp and Bsep. The improvement in Mrp2 levels is of particular interest because this protein transports GSH. As previously mentioned, the biliary secretions of GSH were extensively increased by resveratrol. Accordingly, resveratrol may enhance the biliary secretions of GSH by an Mrp2-mediated acceleration in ANIT elimination. Another substrate of the Mrp2 transporter is conjugated bilirubin. The output of TBIL and DBIL were significantly increased after resveratrol pretreatment (Table 3). These results may be due to the upregulation of Mrp2 by resveratrol. The reduced expression of Mrp3 by resveratrol may also contribute to a decrease in the countertransport of bile acids into the blood, thus reducing bile acids in systemic circulation.

Recent research suggests that the transcriptional regulation of both hepatic bile acids transporters and enzymatic systems involve the nuclear receptors PXR (pregnane $X$ receptor) and FXR (farnesoid $X$ receptor). These nuclear receptors have complementary roles in the regulation of genes involved in lipid and lipoprotein metabolism, including bile acid synthesis and transport. In addition, these proteins are actively involved in the spontaneous adaptive response to cholestasis triggered by the accumulation of bile acids. The receptors aim to reduce the synthesis of these bile acids by increasing the extrahepatic disposal of these potentially toxic compounds ${ }^{[5,37]}$. In our present study, the decreased expression of transporters was accompanied by the decreased expression of FXR and Cyp7a1 after ANIT intoxication; furthermore, resveratrol enhanced the expression of FXR and Cyp7a1. Regulation of the hepatobiliary transporter by resveratrol is poorly defined during cholestasis. It has been reported that the FXR agonist GW4064 can upregulate BESP and Ntcp mRNA expression in the ANIT model of cholestasis ${ }^{[23]}$. We speculate that resveratrol may affect the bile acid transporters in a unique way that differs from the FXR agonists, as no effects on Bsep and Ntcp expression were observed. Previous studies reported that the activation of SIRT1 by resveratrol substantially decreased the acetylated FXR levels in the livers of $o b / o b$ mice. In addition, treatment with resveratrol significantly decreased the Shp mRNA levels and elevated the bile acid pool sizes in mice ${ }^{[38]}$. Our results confirmed that resveratrol enhanced the expression of transporters and enzymes correlated with elevated FXR levels in vivo. These data further support the model that resveratrol exerts additional choleretic effects independent of 
cytoprotection during ANIT-induced acute cholestasis in rats.

In conclusion, the results of this study indicate that resveratrol exerts a preventive effect on ANIT-induced acute liver injury with cholestasis in rats. This effect may be mediated by an attenuation in the levels of bile acids that commonly accumulate following disruptions to transporters and enzymes in the liver tissue or by choleretic and anti-inflammatory actions, leading to an inhibition of neutrophil infiltration.

\section{Abbreviations}

UDCA, ursodeoxycholic acid; ANIT, a-Naphthylisothiocyanate; ALT, alanine aminotransferase; AST, aspartate aminotransferase; ALP, alkaline phosphatase; GGT, ү-glutamyl transpeptidase; TBA, total bile acid; TBIL, total bilirubin; DBIL, direct bilirubin; IBIL, indirect bilirubin; GSH, glutathione; GPx, glutathione peroxidase; MnSOD, manganese superoxide dismutase; MPO, myeloperoxidase; Ntcp, sodium taurocholate cotransporting polypeptide; Bsep, bile salt export pump; Mrp2/3, multidrug-resistant-associated protein 2/3; Cyp7a1, cholesterol 7a-hydroxylase; FXR, farnesoid X receptor.

\section{Acknowledgements}

This study was supported by grants from the National Natural Science Foundation of China (No 81320108029) and the 2011 Program for Excellent Scientific and Technological Innovation Team of Jiangsu Higher Education.

\section{Author contribution}

Tao WANG, Zhen-zhou JIANG, and Lu-yong ZHANG designed the research; Tao WANG, Zhi-xing ZHOU, Li-xin SUN, Xia LI, Zhi-meng XU, and Mi CHEN performed the research; Zhen-zhou JIANG and Tao WANG analyzed the data; Tao WANG and Guo-lin ZHAO wrote the paper.

\section{References}

1 Yamazaki M, Miyake M, Sato H, Masutomi N, Tsutsui N, Adam KP, et al. Perturbation of bile acid homeostasis is an early pathogenesis event of drug induced liver injury in rats. Toxicol Appl Pharmacol 2013; 268: 79-89.

2 Maillette de Buy Wenniger L, Beuers U. Bile salts and cholestasis. Dig Liver Dis 2010; 42: 409-18.

3 Allen $\mathrm{K}$, Jaeschke $\mathrm{H}$, Copple BL. Bile acids induce inflammatory genes in hepatocytes: a novel mechanism of inflammation during obstructive cholestasis. Am J Pathol 2011; 178: 175-86.

4 Schulz S, Schmitt S, Wimmer R, Aichler M, Eisenhofer S, Lichtmannegger J, et al. Progressive stages of mitochondrial destruction caused by cell toxic bile salts. Biochim Biophys Acta 2013; 1828 : 2121-33.

5 Wagner M, Zollner G, Trauner M. Nuclear receptor regulation of the adaptive response of bile acid transporters in cholestasis. Semin Liver Dis 2010; 30: 160-77.

6 Chen F, Ananthanarayanan M, Emre S, Neimark E, Bull LN, Knisely AS, et al. Progressive familial intrahepatic cholestasis, type 1, is associated with decreased farnesoid $X$ receptor activity. Gastroenterology 2004; 126: 756-64.

7 Kosters A, Felix JC, Desai MS, Karpen SJ. Impaired bile acid handling and aggravated liver injury in mice expressing a hepatocyte-specific RXR $\alpha$ variant lacking the DNA-binding domain. J Hepatol 2014; 60:
362-9.

8 Stedman C, Liddle C, Coulter S, Sonoda J, Alvarez JG, Evans RM, et al. Benefit of farnesoid $X$ receptor inhibition in obstructive cholestasis. Proc Natl Acad Sci U S A 2006; 103: 11323-8.

9 Xie Y, Hao HP, Wang H, Wang ZX, Wang GJ. Reversing effects of silybin on TAA-induced hepatic CYP3A dysfunction through PXR regulation. Chin J Nat Med 2013; 11: 645-52.

10 Lagouge M, Argmann C, Gerhart-Hines Z, Meziane H, Lerin C, Daussin $\mathrm{F}$, et al. Resveratrol improves mitochondrial function and protects against metabolic disease by activating SIRT1 and PGC-1alpha. Cell 2006; 127: 1109-22.

11 Wu CF, Yang JY, Wang F. Resveratrol: botanical origin, pharmacological activity and applications. Chin J Nat Med 2013; 11: 1-15.

12 Chan CC, Cheng LY, Lin CL, Huang YH, Lin HC, Lee FY. The protective role of natural phytoalexin resveratrol on inflammation, fibrosis and regeneration in cholestatic liver injury. Mol Nutr Food Res 2011; 55: 1841-9.

13 Hussein MA. Prophylactic effect of resveratrol against ethinylestradiolinduced liver cholestasis. J Med Food 2013; 16: 246-54.

14 Lin TK, Huang LT, Huang YH, Tiao MM, Tang KS, Liou CW. The effect of the red wine polyphenol resveratrol on a rat model of biliary obstructed cholestasis: involvement of anti-apoptotic signalling, mitochondrial biogenesis and the induction of autophagy. Apoptosis 2012; 17: 871-9.

15 Slitt AL, Allen K, Morrone J, Aleksunes LM, Chen C, Maher JM, et al. Regulation of transporter expression in mouse liver, kidney, and intestine during extrahepatic cholestasis. Biochim Biophys Acta 2007; 1768: $637-47$

16 Miyata M, Tozawa A, Otsuka H, Nakamura T, Nagata K, Gonzalez $F J$, et al. Role of farnesoid $X$ receptor in the enhancement of canalicular bile acid output and excretion of unconjugated bile acids: A mechanism for protection against cholic acid-induced liver toxicity. J Pharmacol Exp Ther 2005; 312: 759-66.

17 Plaa, GL, Priestley BG. Intrahepatic cholestasis induced by drugs and chemicals. Pharmacol Rev 1976; 28: 207-73.

18 Zhou ZX, Wang T, Jiang ZZ, Shang J, Liu L, Zhang Y, et al. Beneficial effects of colchicine on 17alpha-ethynylestradiol-induced cholestasis in rats. Arzneimittelforschung 2011; 61: 173-9.

19 Wang J, Jiang Z, Ji J, Li Y, Chen M, Wang Y, et al. Evaluation of hepatotoxicity and cholestasis in rats treated with $\mathrm{EtOH}$ extract of fructus psoraleae. J Ethnopharmacol 2012; 144: 73-81.

20 Dietrich CG, Ottenhoff R, deWaart DR, Oude Elferink RP. Role of MRP2 and GSH in intrahepatic cycling of toxins. Toxicology 2001; 167: 73-81.

21 Ogawa K, Suzuki H, Hirohashi T, Ishikawa T, Meier PJ, Hirose K, et al. Characterization of inducible nature of MRP3 in rat liver. Am J Physiol Gastrointest Liver Physiol 2000; 278: G438-446.

22 Kossor DC, Meunier PC, Handler JA, Sozio RS, Goldstein RS. Temporal relationship of changes in hepatobiliary function and morphology in rats following alpha-naphthylisothiocyanate (ANIT) administration. Toxicol Appl Pharmacol 1993; 119: 108-14.

23 Liu Y, Binz J, Numerick MJ, Dennis S, Luo G, Desai B, et al. Hepatoprotection by the farnesoid X receptor agonist GW4064 in rat models of intra- and extrahepatic cholestasis. J Clin Invest 2003; 112: 1678-87.

24 Giles GI, Tasker KM, Jacob C. Oxidation of biological thiols by highly reactive disulfide-S-oxides. Gen Physiol Biophys 2002; 21: 65-72.

25 Xu SC, Chen YB, Lin H, Pi HF, Zhang NX, Zhao CC, et al. Damage to mtDNA in liver injury of patients with extrahepatic cholestasis: the protective effects of mitochondrial transcription factor A. Free Radic Biol Med 2012; 52: 1543-51. 
26 Arduini A, Serviddio G, Escobar J, Tormos AM, Bellanti F, Viña J, et al. Mitochondrial biogenesis fails in secondary biliary cirrhosis in rats leading to mitochondrial DNA depletion and deletions. Am J Physiol Gastrointest Liver Physiol 2011; 301: G119-27.

27 Dahm $\sqcup$, Schultze AE, Roth RA. An antibody to neutrophils attenuates alpha-naphthylisothiocyanate-induced liver injury. J Pharmacol Exp Ther 1991; 256: 412-20.

28 Hill DA, Jean PA, Roth RA. Bile duct epithelial cells exposed to alphanaphthylisothiocyanate produce a factor that causes neutrophildependent hepatocellular injury in vitro. Toxicol Sci 1999; 47: 11825.

29 Kodali P, Wu P, Lahiji PA, Brown EJ, Maher JJ. ANIT toxicity toward mouse hepatocytes in vivo is mediated primarily by neutrophils via CD18. Am J Physiol Gastrointest Liver Physiol 2006; 291: G355-63.

30 Ohta Y, Kongo M, Sasaki E, Harada N. Change in hepatic antioxidant defense system with liver injury development in rats with a single $\alpha$-naphthylisothiocyanate intoxication. Toxicology 1999; 139: 265-75.

31 Ohta Y, Kongo M, Kishikawa T. Preventive effect of melatonin on the progression of $\alpha$-naphthylisothiocyanate-induced acute liver injury in rats. J Pineal Res 2003; 34: 15-23.

32 Hassan-Khabbar S, Vamy M, Cottart CH, Wendum D, Vibert F, Savouret JF, et al. Protective effect of post-ischemic treatment with trans-resveratrol on cytokine production and neutrophil recruitment by rat liver. Biochimie 2010; 92: 405-10.

33 Paulusma CC, van Geer MA, Evers R, Heijn M, Ottenhoff R, Borst P, et al. Canalicular multispecific organic anion transporter/multidrug resistance protein 2 mediates low-affinity transport of reduced glutathione. Biochem J 1999; 338: 393-401.

34 Slitt AL, Allen K, Morrone J, Aleksunes LM, Chen C, Maher JM, et al. Regulation of transporter expression in mouse liver, kidney, and intestine during extrahepatic cholestasis. Biochim Biophys Acta 2007; 1768: 637-47.

35 Trauner M, Boyer JL. Bile salt transporters: Molecular characterization, function, and regulation. Physiol Rev 2003; 83: 633-71.

36 Guo C, He L, Yao D, A J, Cao B, Ren J, et al. Alpha-naphthylisothiocyanate modulates hepatobiliary transporters in sandwichcultured rat hepatocytes. Toxicol Lett 2014; 224: 93-100.

37 Stahl S, Davies MR, Cook DI, Graham MJ. Nuclear hormone receptordependent regulation of hepatic transporters and their role in the adaptive response in cholestasis. Xenobiotica 2008; 38: 725-77.

38 Kemper JK, Xiao Z, Ponugoti B, Miao J, Fang S, Kanamaluru D, et al. FXR acetylation is normally dynamically regulated by p300 and SIRT1 but constitutively elevated in metabolic disease states. Cell Metab 2009; 10: 392-40. 